\title{
Toward an Endovascular Internal Carotid Artery Classification System
}

\author{
M. Shapiro, T. Becske, H.A. Riina, E. Raz, D. Zumofen, J.J. Jafar, P.P. Huang, and P.K. Nelson
}

\begin{abstract}
SUMMARY: Does the world need another ICA classification scheme? We believe so. The purpose of proposed angiography-driven classification is to optimize description of the carotid artery from the endovascular perspective. A review of existing, predominantly surgically-driven classifications is performed, and a new scheme, based on the study of NYU aneurysm angiographic and cross-sectional databases is proposed. Seven segments - cervical, petrous, cavernous, paraophthlamic, posterior communicating, choroidal, and terminus - are named. This nomenclature recognizes intrinsic uncertainty in precise angiographic and cross-sectional localization of aneurysms adjacent to the dural rings, regarding all lesions distal to the cavernous segment as potentially intradural. Rather than subdividing various transitional, ophthalmic, and hypophyseal aneurysm subtypes, as necessitated by their varied surgical approaches and risks, the proposed classification emphasizes their common endovascular treatment features, while recognizing that many complex, trans-segmental, and fusiform aneurysms not readily classifiable into presently available, saccular aneurysm-driven schemes, are being increasingly addressed by endovascular means. We believe this classification may find utility in standardizing nomenclature for outcome tracking, treatment trials and physician communication.
\end{abstract}

$\mathbf{U}^{\prime}$

ntil now, the ICA was subdivided into various segments, primarily on the basis of surgical considerations. Fischer's original classification (Fig 1), ${ }^{1}$ with $\mathrm{C} 1-\mathrm{C} 5$ segments, in retrograde fashion, addressed the then-existing need for localization of regional pathology on the basis of mass effect on the ICA, as assessed by early angiographic imaging. It remained in widespread use until 1981, when Gibo et $\mathrm{al}^{2}$ in a report detailing superb dissections of the supraclinoid ICA designed to specifically address aneurysm considerations, introduced an antegrade alphanumeric system from C1-C4 (Fig 2). The extracranial ICA was recognized as the $\mathrm{C} 1$ segment, and extradural ICA was subdivided into petrous $(\mathrm{C} 2)$ and cavernous $(\mathrm{C} 3)$ segments, terminating at the distal dural ring. The entire intradural ICA was classified as supraclinoid (C4), with ophthalmic, communicating, and choroidal subsegments (Fig 2). Subsequently, a modified Fischer classification was proposed by Bouthillier et $\mathrm{al}^{3}$ in 1996, which became known

Received February 28, 2013; accepted after revision May 11.

From the Department of Radiology (M.S., T.B., H.A.R., E.R., D.Z., P.K.N.), Bernard and Irene Schwartz Neurointerventional Radiology Section; and Departments of Neurology (M.S., T.B.), and Neurosurgery (H.A.R., D.Z., J.J.J., P.P.H., P.K.N.), NYU Langone Medical Center, New York, New York.

Please address correspondence to Maksim Shapiro, MD, Departments of Radiology and Neurology, Bernard and Irene Schwartz Neurointerventional Radiology Section, NYU Langone Medical Center, 660 First Ave, 7th Floor, New York, NY 10016; e-mail: shapim06@med.nyu.edu

http://dx.doi.org/10.3174/ajnr.A3666 as the Bouthillier classification (Fig 3). Although substantially different from Fischer's, it also retained an antegrade alphanumeric designation of C1-C6 segments. Bouthillier introduced the new lacerum and, most importantly, clinoid segments, thereby formally recognizing a particularly complex region of carotid anatomy as it transitions from intracavernous to intradural spaces. Anatomic interest in this area was driven by refinement in microsurgical techniques, which allowed for the possibility of access to aneurysms of this paraophthalmic-paracliniod region, albeit with mixed results. While Bouthillier's classification gained wide and enduring acceptance, new systems were proposed to address its perceived shortcomings. Ziyal et $\mathrm{al}^{4}$ advocated a classification (Fig 4) that dispensed with Bouthillier's lacerum segment, arguing on the basis of detailed microsurgical dissections that relationships between the carotid artery, foramen lacerum, petrolingual ligament, and proximal cavernous sinus left no room for a distinct lacerum segment. ${ }^{5}$ The ophthalmic segment was also discarded in favor of the cisternal segment because of variability in the index artery origin. ${ }^{4}$ Additional, surgically driven, subsegmental classifications, addressing primarily the paraclinoid and ophthalmic regions, were proposed, incorporating evolving surgical approaches in this challenging area., ${ }^{2,6-11}$

A radically different approach was taken by Lasjaunias et $\mathrm{al}^{12,13}$ (Fig 5), emphasizing embryologic origins of various ICA segments. The system was part of an all-encompassing, conceptually brilliant embryologic framework, addressing all 


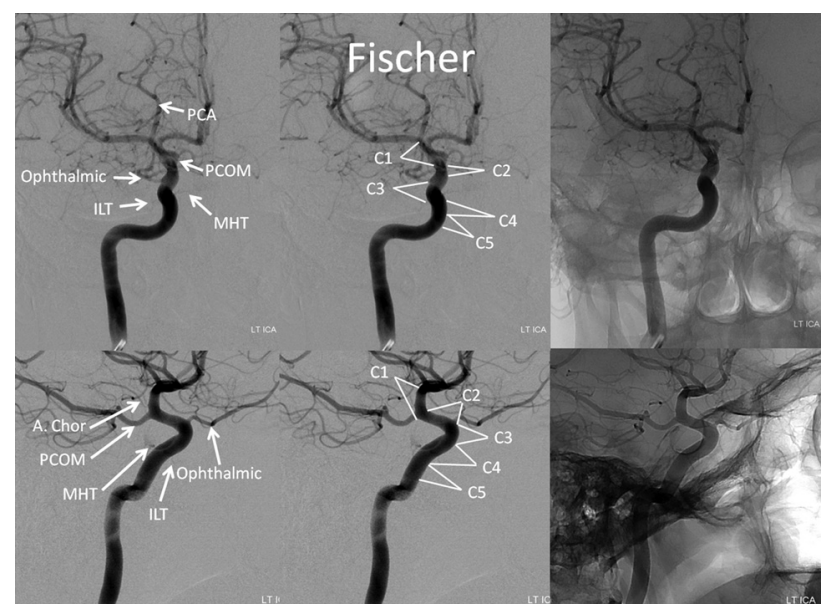

FIG 1. Frontal and lateral projection views of left ICA injection, depicting Fischer ICA nomenclature, including relevant osseous and branch relationships.

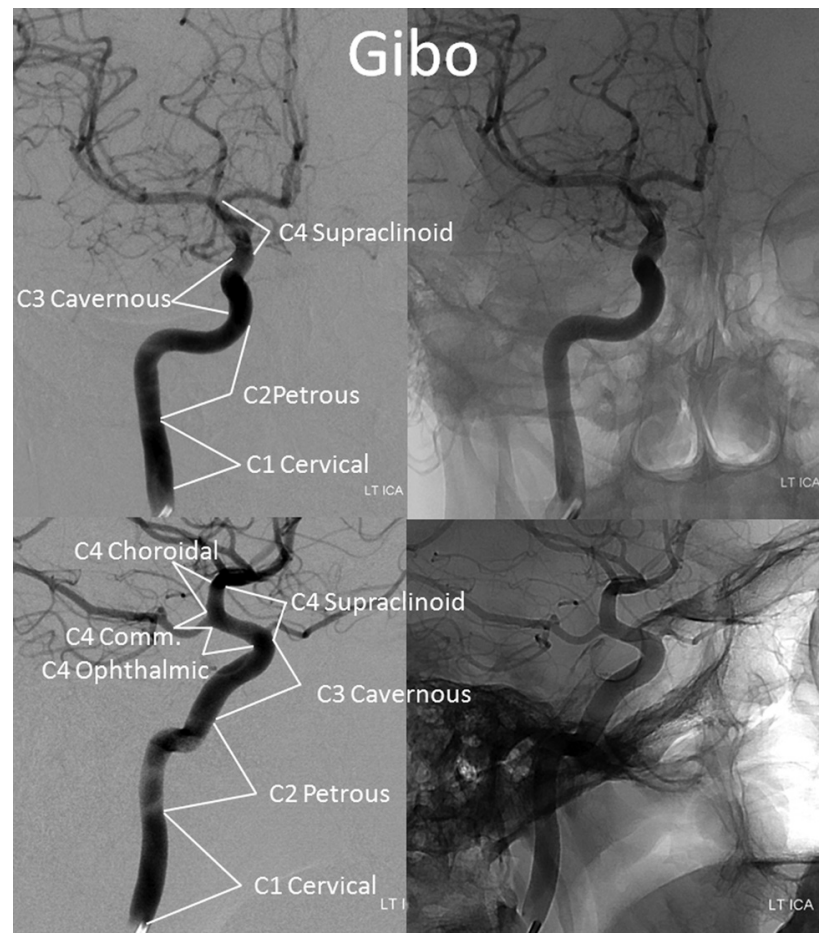

FIG 2. Gibo classification. The C4 supraclinoid segment is subdivided into ophthalmic, communicating, and choroidal subsegments.

manner of cerebrovascular development and variation, such as segmental ICA agenesis and reconstitution, the aberrant ICA, dorsal and ventral ophthalmic origin, and the primitive maxillary artery, to name a few. This unique view was derived from neither surgical nor endovascular experience and as such was not specifically designed for existing microsurgical or thenemerging endovascular considerations. Although the authors introduced a correlation between the embryologic segments and external anatomic landmarks, illustrated in Fig 5, the system did not achieve widespread use.

Our own view of the ICA, crystallized in the currently proposed scheme (Fig 6), emerged as a product of decades-long experience in angiographic and cross-sectional study of vari-

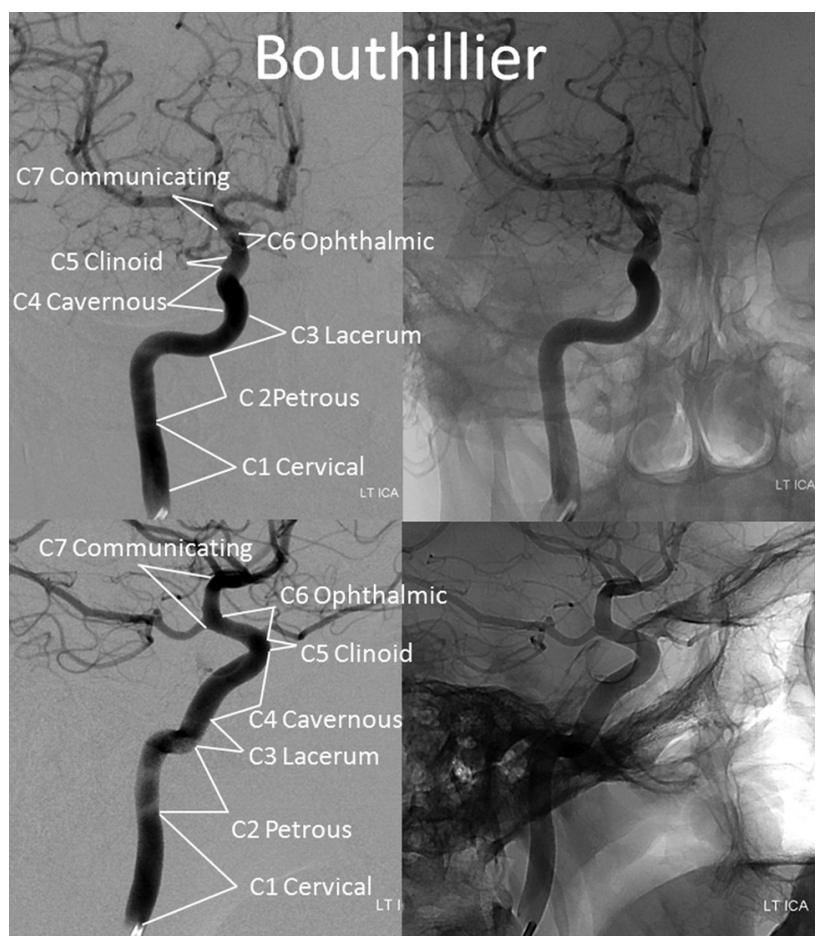

FIG 3. Bouthillier classification.

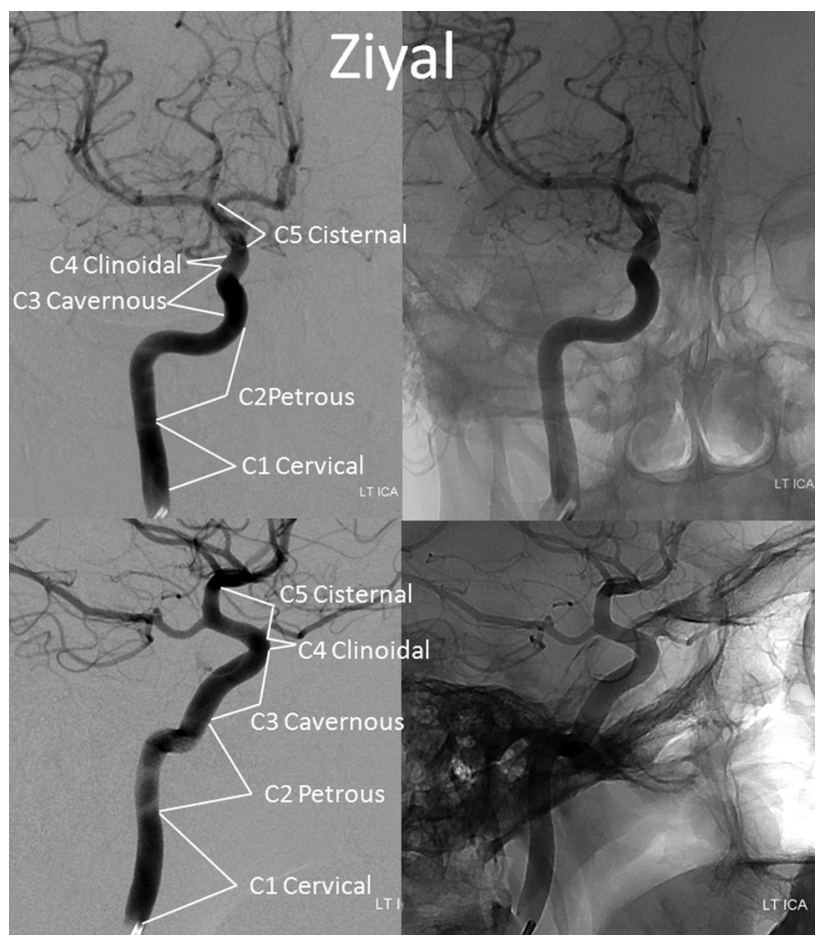

FIG 4. Ziyal classification.

ous ICA aneurysms and subsequent treatment-centered discussions. During this process, we have naturally gravitated toward certain descriptive terms, which seem to transmit most succinctly both anatomic information and subjective implications related to treatment considerations in a multidisciplinary forum, recognizing intrinsic uncertainties between angiographic and microsurgical landmarks. Concurrent refinement 


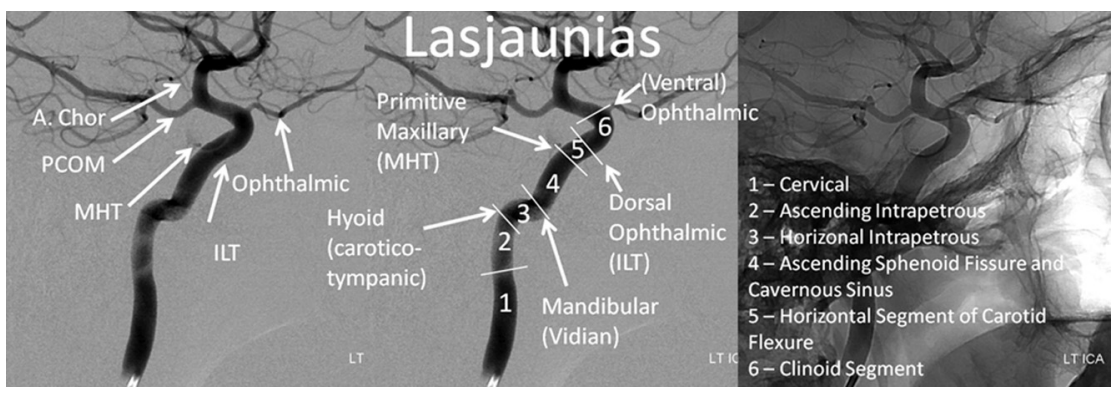

FIG 5. Lasjaunias and Santoyo-Vazquez, embryology-based classification.

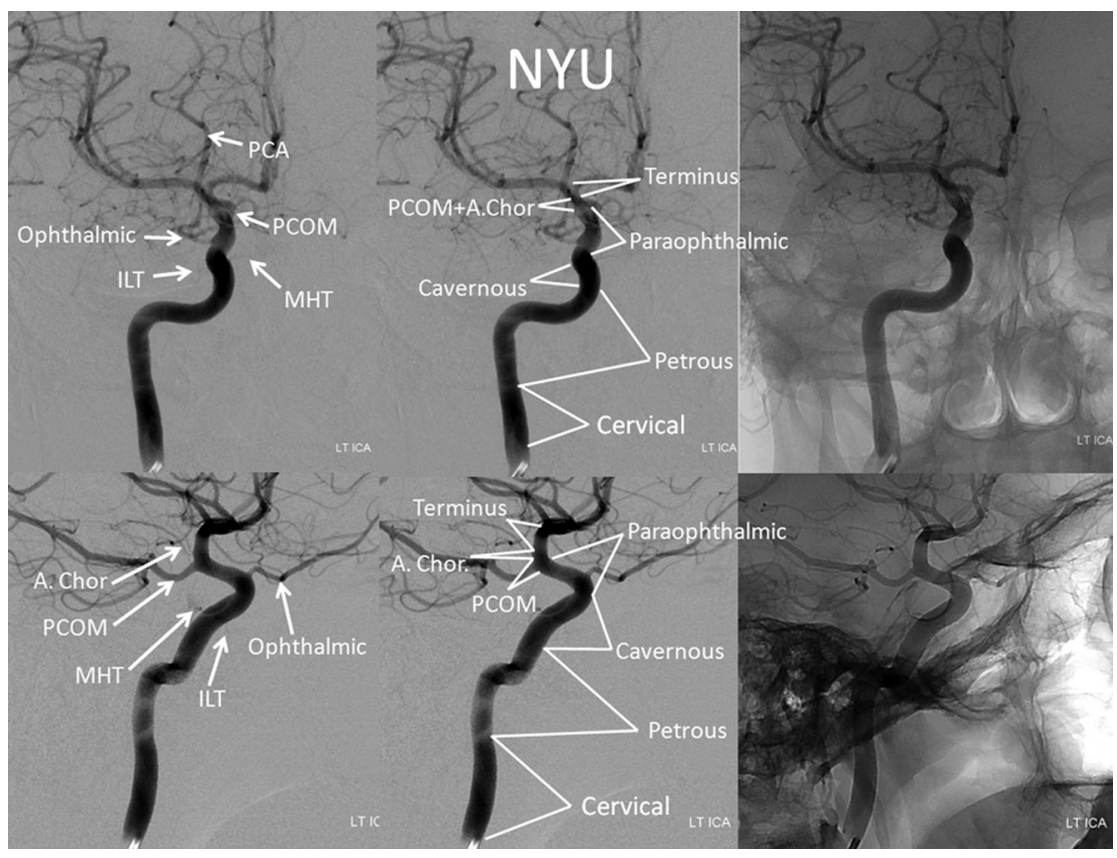

FIG 6. Proposed classification.

in endovascular techniques, most recently exemplified by development of endoluminal (flow diversion) methods, has meanwhile transformed cerebrovascular approaches to carotid siphon aneurysms, placing increasing emphasis on development of an ICA classification system particularly relevant to endovascular factors. ${ }^{14}$ The improved capability to definitively treat larger and increasingly complex ICA aneurysms and fusiform segments of the artery highlights the need for a conceptually flexible classification that suitably describes the transsegmental nature of many such formidable lesions, which frequently extend across various embryologically neutral, landmark-based segmental boundaries.

\section{MATERIALS AND METHODS}

A literature review of existing ICA classification systems and related works was conducted. Cerebral angiograms and crosssectional studies performed at the New York University Langone Medical Center and Bellevue Health and Hospitals Center and outside studies forwarded for consultation were continuously reviewed and discussed at our multidisciplinary conference. Patterns of aneurysm formation and growth were studied as a pathophysiologic guide to a clinically useful classification system.

\section{RESULTS}

The proposed system is presented in Fig 6. In this nomenclature, 7 segments are described-cervical, petrous, cavernous, paraophthalmic, posterior communicating, anterior choroidal, and terminus-without alphanumeric correspondence.

The cervical segment is identical to that of Bouthillier and Ziyal, extending from cervical carotid bifurcation to the proximal margin of the carotid canal. Variations such as aberrant carotid artery (ie, ascending pharyngeal artery reconstitution of petrous carotid artery) can be described on their own terms.

The petrous segment is analogous to that of Ziyal, and, combining Bouthillier's petrous and lacerum segments, spans the ICA within the temporal bone and partially above the foramen lacerum, terminating at the petrolingual ligament. It contains the rarely visible (probably caused by temporal bone thickness rather than actual absence) caroticotympanic branch and the more consistently demonstrated vidian branches. The precise location of the petrolingual ligament is not angiographically determinable, and the anterior aspect of the petrous ridge can be used as a landmark. We chose to dispense with a distinct lacerum segment, in agreement with Ziyal, for 2 reasons. First, the preponderance of anatomic information argues against its unique character, ${ }^{5,15}$ whereas the petrolingual ligament best defines carotid transition into the cavernous segment. Second, from a pathophysiologic standpoint, among the nontraumatic temporal bone aneurysms evaluated by our group that involved the lacerum portion of the ICA, none were focal; all were fusiform lesions extending into the adjacent petrous or cavernous segments, rendering the distinction between Bouthillier's petrous and lacerum segments clinically neutral (Fig 7).

The cavernous segment extends from the estimated location of distal margin of the petrolingual ligament to the estimated location of the proximal dural ring. We recognize that neither proximal nor, more importantly, distal cavernous segment boundaries, can be reliably established on an angiographic or transaxial basis - a recurrent theme with regard to segmentation on the basis of external anatomic landmarks. Additionally, the morphology of both cavernous sinus and cavernous ICA are highly variable, with important endovascular implications. Broadly speaking, a proximal ascending segment, proximal (posterior) genu, horizontal segment, distal (anterior) genu, and distal ascending segment can 


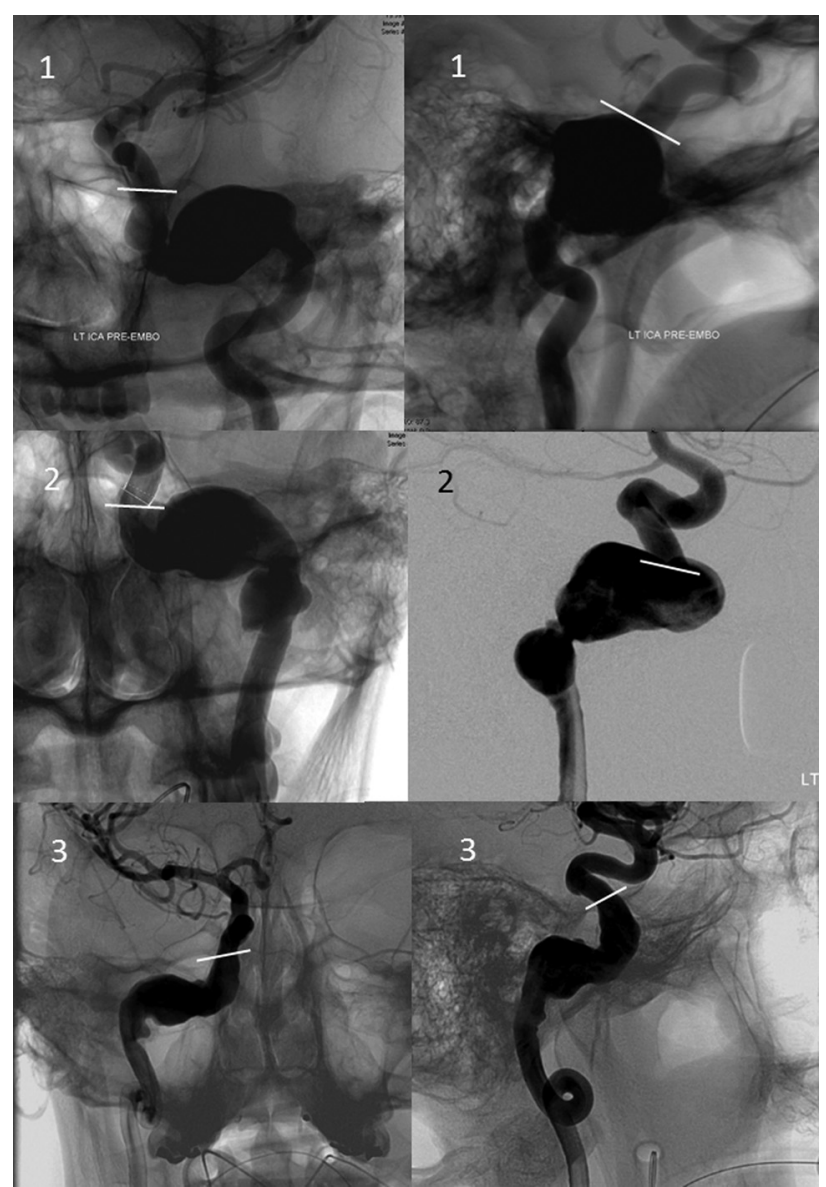

FIG 7. Frontal, transorbital (upper left image), and lateral views of 3 petrous segment aneurysms, demonstrating their extension into the vertical segment above the foramen lacerum, terminating adjacent to the superior margin of the petrous apex (white lines), corresponding to estimated location of the petrolingual ligament.

be described. The meningohypophyseal trunk or its component branches originate from the posterior genu, whereas the inferolateral trunk is most commonly found arising from the horizontal segment. It is interesting to note that aneurysms of the cavernous segment, regardless of size and morphology, appear to spare the proximal vertical portion, which extends from the petrolingual ligament to the proximal genu, even though this subsegment is anatomically located within the posterior compartment of the cavernous sinus (Fig 8). The reasons for this are unclear: an embryologic mechanism could be suggested from the Lasjaunias system, corresponding to segment 4 in Fig 5. The uncertainty in localizing the distal boundary of the cavernous segment is formally recognized in the next, encompassing paraophthalmic segment.

The paraophthalmic segment extends from the estimated distal border of the cavernous segment to the ostium of the posterior communicating artery, with all of its aneurysms regarded as clearly or potentially intradural. Our choice of this generalized term follows from 2 primary considerations. First, we formally accept the intrinsic uncertainly in angiographic or cross-sectional definition of traditional segmental boundaries (particularly relevant to delineation of the clinoid segment). Second, we propose that endovascular methods, in contrast to surgical ones, increas-

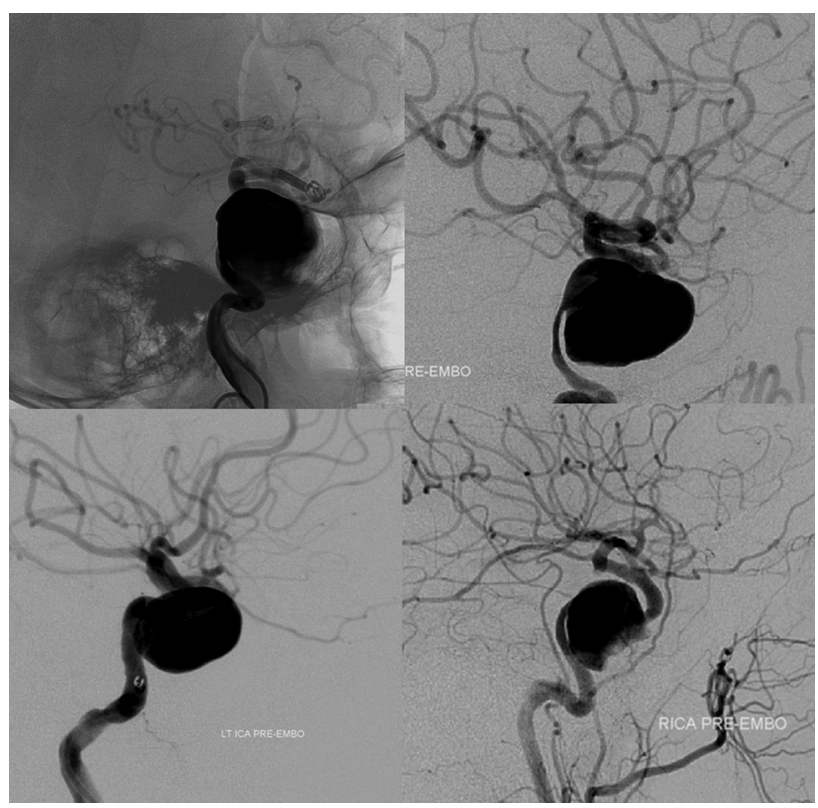

FIG 8. Four fusiform cavernous segment aneurysms, all sparing the proximal vertical subsegment of the cavernous ICA.

ingly tend to unite rather than divide treatment considerations of the various aneurysms arising from traditionally defined clinoid, ophthalmic, and superior hypophyseal segments.

Proximally, the paraophthalmic segment incorporates Bouthillier's clinoid segment, which can be microsurgically localized between the proximal and distal dural rings. ${ }^{3,16}$ This assimilation formally recognizes that neither location nor status of either ring is reliably demonstrable with presently available angiographic or transaxial imaging modalities. Attempts to define the position of a distal dural ring, by use of CT, MR imaging, or angiography, ${ }^{6,17-21}$ are limited by 2 key considerations. First, none of the clinically available imaging modalities can actually depict the distal dural ring itself; attempts to define its location instead rely on indirect morphometric and landmark relationships. As such, these methods become quite vulnerable to a high degree of local anatomic variation. No attempt has been made, so far as we know, to demonstrate the more nebulous and less clinically significant proximal ring. Second, and more importantly, regional anatomy is extensively modified by disease (aneurysm growth), which distorts landmarks by destroying or distorting adjacent tissues (dural ring dehiscence). For these reasons, short of surgical exposure, the exact state of affairs in this region cannot be determined by any currently available imaging technique. Finally, the subpopulation of larger and more complex aneurysms, which are increasingly addressed by endovascular methods, frequently transcend established segmental boundaries, leading to uncertainty as to whether a given lesion is partially or fully, primarily or secondarily, intradural (Fig 9). Our position is to consider all aneurysms distal to the anterior cavernous carotid artery genu as potentially intradural.

The endovascular considerations involved in treatment of proximal paraophthalmic segment aneurysms are separate and distinct from well-established microsurgical concerns of exposing the clinoid segment and cave aneurysms, ${ }^{7,16}$ which are different 


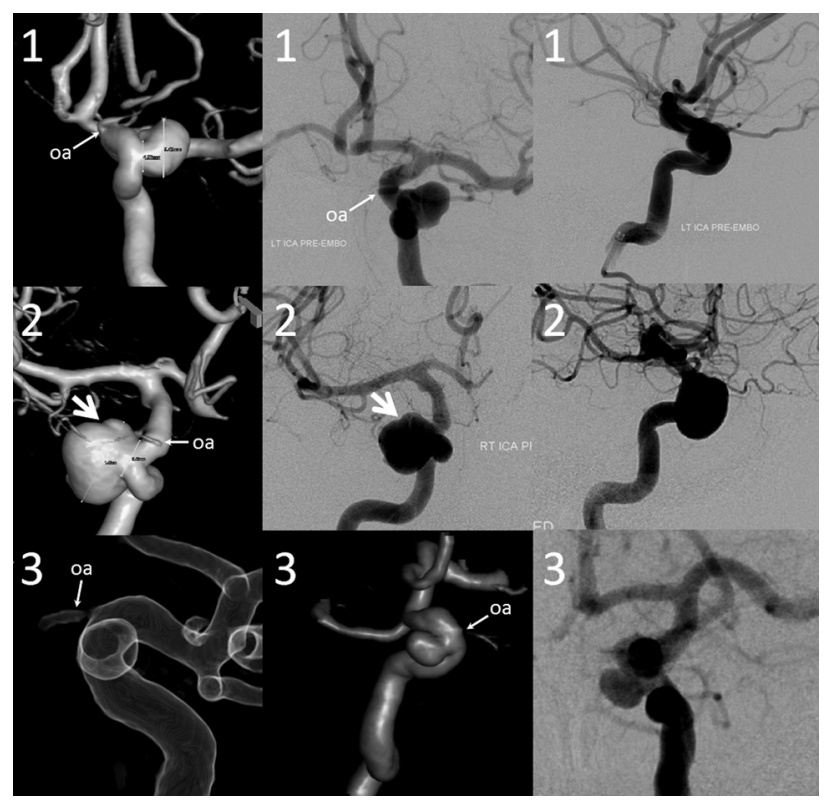

FIG 9. Three-dimensional DSA, frontal, and lateral projection views of 3 proximal paraophthalmic aneurysms, which would traditionally be considered in the clinoid (1,2) and cave (3) families. An oblique indentation (arrow) of aneurysm 2 probably defines the dural ring, suggesting intradural extension.

from surgical approaches to various ophthalmic or superior hypophyseal region aneurysms. No such critical distinctions are relevant in the endovascular arena, in which the most important point is to decide whether a given aneurysm is extradural or intradural. In consequence, all intradural ICA subsegments proximal to the posterior communicating artery are consolidated into the paraophthalmic segment. This area is home to particularly varied and complex aneurysms, in which our experience suggests a lower baseline interobserver agreement as to their classification, becoming progressively more difficult with larger and frankly trans-segmental lesions (Fig 10). The proposed nomenclature also reflects the angiographic limitations of identifying the superior hypophyseal arteries, which serve to define their aneurysms in microsurgical dissections. These arteries are small and have a variable, typically medial origin along a comparatively long segment of the ICA. They tend to be superimposed on the parent ICA in lateral projections and therefore are rarely demonstrable on angiography. Furthermore, the amalgamation of all aneurysms proximal to the posterior communicating artery into the paraophthalmic segment encompasses infrequent but important aneurysms not necessarily associated with the superior hypophyseal arteries (such as treacherous ventral or dorsal blister lesions).

An awkward situation for use of the "paraophthalmic" term is created when the ophthalmic artery does not arise from its "classic" location in the very proximal intradural ICA. On the other hand, greater flexibility is now afforded for the location of ophthalmic artery ostium, with its intradural (traditional clinoid segment) variants remaining within the paraophthalmic segment. Our feeling is that other potential sites of orbital supply, which include the dorsal ophthalmic artery arising in the adult from the

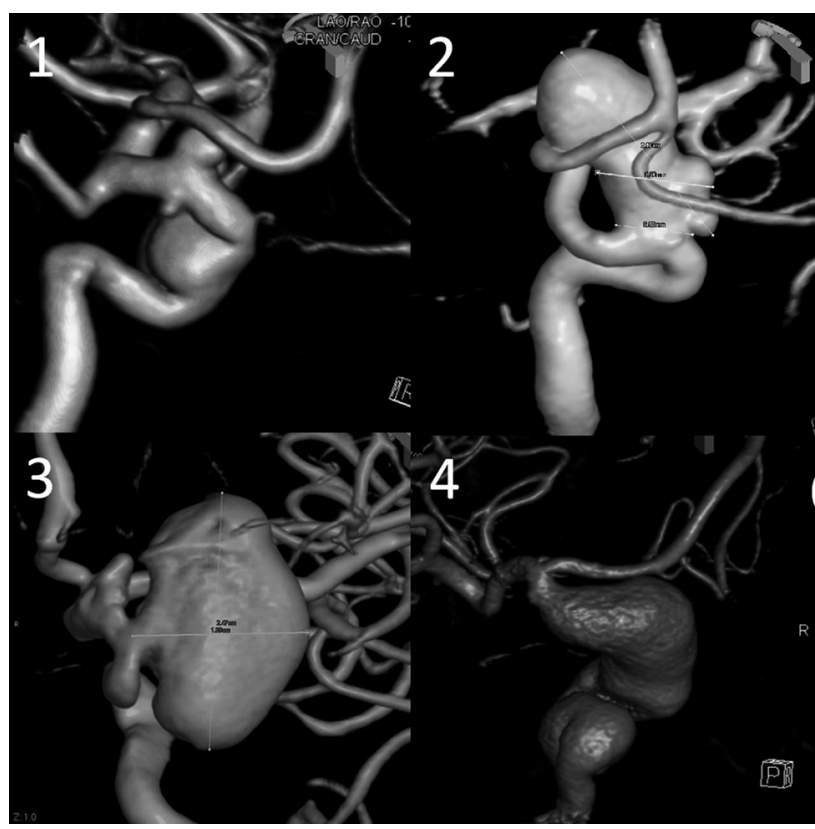

FIG 10. Various 3D DSA examples of segmental ICA dysplasia. In traditional classifications, case 1 may be described as 3 separate aneurysms-distal cavernous (or transitional) and superior- and inferiorpointing superior hypophyseal aneurysms. We consider all of these as focal expressions of disease affecting the entire paraophthalmic segment. Case 2 may be considered as a discrete carotid-ophthalmic aneurysm; notice, however, the irregular, expanded morphology of the parent vessel below the "neck." Case 3-apparently separate clinoid and ophthalmic aneurysms - can also be viewed as segmental dysplasia with a single aneurysm separated by the anterior clinoid process. Case 4 is a giant fusiform aneurysm spanning the entire carotid artery from cavernous to terminus segments.

inferolateral trunk, external carotid origin particularly from the middle meningeal artery (meningo-ophthalmic variant), and ventral ophthalmic variants arising from the anterior cerebral artery are sufficiently uncommon that descriptions of ICA pathology in this region may be customized accordingly, with minimal detriment to the system as a whole. The issue may be addressed by adoption of another term that makes no mention of the ophthalmic artery-as reflected in the Ziyal classification through the "cisternal" segment ${ }^{4}$; our preference is to emphasize the more common anatomic arrangement, made further flexible by extension of the paraophthalmic segment into the traditional clinoid region.

The posterior communicating segment extends from the proximal ostium of the posterior communicating artery to the anterior choroidal artery. A separate category for the posterior communicating segment recognizes the unique epidemiologic characteristics of its aneurysms. ${ }^{22}$ Furthermore, in contrast to preceding segments, the angiographic boundaries of this segment can be adequately defined under most circumstances. Continued vernacular description of aneurysms arising from the posterior communicating segment of the ICA as simply "posterior communicating" is fully acceptable because there is no uncertainty about what is meant by either name. Finally, both endosaccular and endoluminal treatment considerations-related to preservation, sacrifice, or jailing of the posterior communicating artery, as well 
as issues of posttreatment recurrence and its management-are distinct from those of the subsequent choroidal segment.

The anterior choroidal segment consists of the ICA adjacent to the choroidal ostium and includes aneurysms recognized in the vernacular description as anterior choroidal. The distinct issues of maintaining anterior choroidal patency and risk stratification in cases of its compromise (intentional or not) are germane to both surgical and endovascular modalities.

The terminus segment extends from beyond the choroidal ostium to the carotid bifurcation and incorporates aneurysms arising from the carotid bifurcation. It contains a variable number of perforator vessels to the anterior perforated substance, ${ }^{23}$ which, along with anterior choroidal branches, participate extensively in Moyamoya-type collateral reconstitution of the MCA and anterior cerebral artery. We believe that both clinical and management considerations of terminus aneurysms are sufficiently different from the choroidal type and thus merit placement in a separate category.

\section{DISCUSSION}

The proposed angiographic classification seeks to define each vascular segment in terms of its distinct angiographic and endovascular considerations. This leads to both amalgamation of commonly defined segments (clinoid, ophthalmic, and hypophyseal) and subdivision in the postcommunicating region. Cervical segment pathology is widely recognized as separate from cranial lesions. Petrous segment aneurysms, both traumatic and nontraumatic, likewise represent a unique set of pathology, as do various cavernous aneurysms. The full spectrum of paraophthalmic aneurysms, inclusive of the large and complex lesions that remained on the fringes of surgical classifications driven by more discrete saccular aneurysms, are thought to represent a sufficiently unified group from an endovascular perspective. The posterior communicating, anterior choroidal, and terminus segment aneurysms are all associated with their particular clinical and technical issues.

Our improving ability to definitively treat the increasingly complex and trans-segmental aneurysms requires adoption of a more flexible system, capable of comfortably accommodating these lesions within a descriptive framework. We hope that the proposed nomenclature will increase interobserver agreement in classification of ICA lesions, particularly desirable in epidemiologic and other research settings in addition to the clinical setting. Finally, we formally dispensed with numeric classification in favor of purely descriptive terms to minimize confusion with various already-existing schemes.

We remain acutely cognizant that despite our sincere effort, all landmark-based classification systems, including ours, remain intrinsically suboptimal when applied to description of increasingly complex, trans-segmental aneurysms. Therefore, in closing, we wish to re-emphasize that all classifications have a priori limitations and are most useful when they strike a balance between utility, accuracy, and flexibility-particularly stressing the latter quality-as demanded by the varied nature of the pathologies these systems are designed to address.

\section{CONCLUSIONS}

We propose a new, angiographically based classification system of the ICA, from cervical to intracranial bifurcations, to better serve the needs of present-day endovascular clinical and research efforts.

Disclosures: Tibor Becske-UNRELATED: Consultancy: Covidien, Comments: clinical proctorships related to Pipeline Embolization Device; Maksim Shapiro-UNRELATED: Consultancy: Covidien, Comments: clinical proctorships related to Pipeline Embolization Device; Howard Riina-UNRELATED: Board Membership: Reach Bionics; Grants/Grants Pending: Tomorrow Foundation*; Patents (planned, pending, or issued): None relevant to this area of work; Stock/Stock Options: Reach Bionics. Peter Nelson—UNRELATED: Consultancy: Covidien, Comments: Clinical proctorships related to Pipeline Embolization Device; Royalties: Stryker, ${ }^{*}$ Comments: Royalties related to Neuroform and Wingspan Stents ( ${ }^{*}$ money paid to institution).

\section{REFERENCES}

1. Fischer E. Die Lageabweichungen der vorderen Hirnarterie im Gefässbild. Zentralbl Neurochir 1938;3:300-13

2. Gibo H, Lenkey C, Rhoton AL. Microsurgical anatomy of the supraclinoid portion of the internal carotid artery. J Neurosurg 1981;55:560-74

3. Bouthillier A, van Loveren HR, Keller JT. Segments of the internal carotid artery: a new classification. Neurosurgery 1996;38:425-32

4. Ziyal IM, Ozgen T, Sekhar LN, et al. Proposed classification of segments of the internal carotid artery: anatomical study with angiographical interpretation. Neurol Med Chir (Tokyo) 2005;45:184-90

5. Ziyal IM, Salas E, Wright DC, et al. The petrolingual ligament: the anatomy and surgical exposure of the posterolateral landmark of the cavernous sinus. Acta Neurochir (Wien) 1998;140:201-04

6. Beretta $F$. The paraclinoid aneurysms and the distal dural ring: a new classification. J Neurosurg Sci 2004;48:161-75

7. Kobayashi S, Kyoshima K, Gibo H, et al. Carotid cave aneurysms of the internal carotid artery. J Neurosurg 1989;70:216-21

8. Kiris T, Sankhla S, Lawton M, et al. Microsurgical anatomy of the cavernous sinus. Barrow Quarterly 1996;12:1-7

9. Day AL. Aneurysms of the ophthalmic segment: a clinical and anatomical analysis. J Neurosurg 1990;72:677-91

10. Barami K, Hernandez VS, Diaz FG, et al. Paraclinoid carotid aneurysms: surgical management, complications, and outcome based on a new classification scheme. Skull Base 2003;13:31-41

11. De Jesús O, Sekhar LN, Riedel CJ. Clinoid and paraclinoid aneurysms: surgical anatomy, operative techniques, and outcome. Surg Neurol 1999;51:477-87

12. Lasjaunias PL, Berenstein A, Raybaud C. Surgical Neuroangiography. Berlin/New York: Springer-Verlag; 1987

13. Lasjaunias $P$, Santoyo-Vazquez A. Segmental agenesis of the internal carotid artery: angiographic aspects with embryological discussion. Anat Clin 1984;6:133-41

14. Fiorella D, Woo HH, Albuquerque FC, et al. Definitive reconstruction of circumferential, fusiform intracranial aneurysms with the Pipeline embolization device. Neurosurgery 2008;62:1115-20

15. Ferreira M, Feiz-Erfan I, Deshmukh P, et al. Microsurgical anatomy of the anterior cerebral circulation, part 1: the infraclinoid internal carotid artery. Barrow Quarterly; 2002;18:1-7

16. Oikawa S, Kyoshima K, Kobayashi S. Surgical anatomy of the juxtadural ring area. J Neurosurg 1998;89:250-54

17. Thines L, Delmaire C, Le Gars D, et al. MRI localization of paraclinoid carotid aneurysms. J Neuroradiol 2006;33:115-20

18. Thines L, Gauvrit JY, Leclerc X, et al. Usefulness of MR imaging for the assessment of nonophthalmic paraclinoid aneurysms. AJNR Am J Neuroradiol 2008;29:125-29

19. Thines L, Lee SK, Dehdashti AR, et al. Direct imaging of the distal dural ring and paraclinoid internal carotid artery aneurysms with

AJNR Am J Neuroradiol 35:230-36 Feb 2014 www.ajnr.org

235 
high-resolution T2 turbo-spin echo technique at 3-T magnetic resonance imaging. Neurosurgery 2009;64:1059-64

20. Hirai T, Kai Y, Morioka M, et al. Differentiation between paraclinoid and cavernous sinus aneurysms with contrast-enhanced 3D constructive interference in steady-state MR imaging. AJNR Am J Neuroradiol 2008;29:130-33

21. Lee N, Jung JY, Huh SK, et al. Distinction between intradural and extradural aneurysms involving the paraclinoid internal carotid ar- tery with $\mathrm{T} 2$-weighted three-dimensional fast spin-echo magnetic resonance imaging. J Korean Neurosurg Soc 2010;47:437-41

22. International Study of Unruptured Intracranial Aneurysms Investigators. Unruptured intracranial aneurysms: risk of rupture and risks of surgical intervention. $N$ Engl $J$ Med 1998;339:1725-33

23. Rhoton AL. The supratentorial arteries. Neurosurgery 2002;51:S53120 